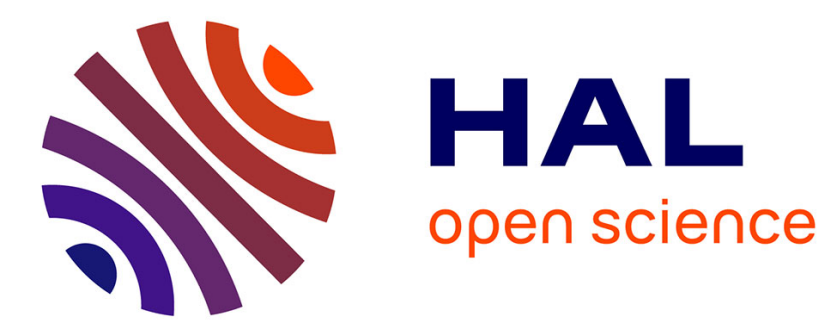

\title{
Anisotropy-guided enantiomeric enhancement in alanine using far-UV circularly polarized light
}

Cornelia Meinert, Patrick Cassam-Chenaï, Nykola C. Jones, Laurent Nahon, Søren V. Hoffmann, Uwe J. Meierhenrich

\section{- To cite this version:}

Cornelia Meinert, Patrick Cassam-Chenaï, Nykola C. Jones, Laurent Nahon, Søren V. Hoffmann, et al.. Anisotropy-guided enantiomeric enhancement in alanine using far-UV circularly polarized light. Origins of Life and Evolution of Biospheres, 2015, 45 (1-2), p1-13. 10.1007/s11084-015-9413-x . hal01128966

\section{HAL Id: hal-01128966 \\ https://hal.science/hal-01128966}

Submitted on 10 Mar 2015

HAL is a multi-disciplinary open access archive for the deposit and dissemination of scientific research documents, whether they are published or not. The documents may come from teaching and research institutions in France or abroad, or from public or private research centers.
L'archive ouverte pluridisciplinaire HAL, est destinée au dépôt et à la diffusion de documents scientifiques de niveau recherche, publiés ou non, émanant des établissements d'enseignement et de recherche français ou étrangers, des laboratoires publics ou privés.

\section{(ㅇ)(1) $\$$}

Distributed under a Creative Commons Attribution - NonCommercial - NoDerivatives $\mid 4.0$ 


\title{
Anisotropy-guided enantiomeric enhancement in alanine using far-UV circularly polarized light
}

Cornelia Meinert*, Patrick Cassam-Chenaï, Nykola C. Jones, Laurent Nahon, Søren V. Hoffmann, Uwe J. Meierhenrich

Dedicated to Professor Edward Rubenstein on the occasion of his $90^{\text {th }}$ birthday

Dr. C. Meinert, Prof. Dr. U. J. Meierhenrich

Institut de Chimie de Nice ICN, CNRS UMR 7272

Université de Nice Sophia Antipolis, 06108 Nice (France)

Dr. P. Cassam-Chenaï

Laboratoire J. A. Dieudonné, CNRS UMR 7351

Université de Nice Sophia Antipolis (France)

Dr. N. C. Jones, Dr. S. V. Hoffmann

Institute for Storage Ring Facilities (ISA), Dept. of Physics and Astronomy

Aarhus University, 8000 Aarhus C (Denmark)

Dr. L. Nahon,

Synchrotron SOLEIL,

91192 Gif-sur-Yvette (France)

Corresponding author:

Email: cornelia.meinert@unice.fr; Tel: +33 492076136; Fax: +33 492076151

\begin{abstract}
All life on Earth is characterized by its asymmetry - both the genetic material and proteins are composed of homochiral monomers. Understanding how this molecular asymmetry initially arose is a key question related to the origins of life. Cometary ice simulations, L-enantiomeric enriched amino acids in meteorites and the detection of circularly polarized electromagnetic radiation in star-forming regions point to a possible interstellar/protostellar generation of stereochemical asymmetry. Based upon our recently recorded anisotropy spectra $g(\lambda)$ of amino acids in the vacuum-UV range, we subjected amorphous films of racemic ${ }^{13} \mathrm{C}$-alanine to far-UV circularly polarized synchrotron radiation to probe the asymmetric photon-molecule interaction under interstellar conditions. Optical purities of up to $4 \%$ were reached, which correlate with our theoretical predictions. Importantly, we show that chiral symmetry breaking using circularly polarized light is dependent on both the helicity and the wavelength of incident light. In order to predict such stereocontrol, time-dependent density functional theory was used to calculate anisotropy spectra. The calculated anisotropy spectra show good agreement with the experimental ones. The European Space Agency's Rosetta mission, which successfully landed Philae on comet 67P/Churyumov-Gerasimenko on 12 November 2014, will investigate the configuration of chiral compounds and thereby obtain data that are to be interpreted in the context of the results presented here.
\end{abstract}

\section{Keywords}

amino acids; anisotropy; homochirality; asymmetric photolysis; origins of life; chiral photons 


\section{Introduction}

One of the most striking aspects of nature is that it is 'one handed' - out of two possible mirror images, biological molecules almost exclusively exist in just one form. That biopolymers evolved to use L-amino acids in proteins and D-sugars in nucleic acids is thought to be an essential aspect of the evolution of life on Earth (Brack et al. 1998). Moreover, there is considerable evidence that forming these biopolymers from homochiral material is necessary for their function. One Hypothesis for the evolutionary origin of this strict stereochemical selection is the asymmetric photochemistry model (Evans et al. 2012; Meinert et al. 2011) by which circularly polarized (CP) photons induce an enantiomeric excess (ee) in chiral organic molecules via asymmetric photolysis. The transfer of a distinct chirality from chiral photons to organic molecules is assumed to have occurred in environments of interstellar molecular clouds. This model is supported by the observation of CP light in various star-forming regions (Bailey et al. 1998; Kwon et al. 2014) and the identification of L-enantiomerically enriched $\left(e e_{\mathrm{L}}\right)$ amino acids in carbonaceous chondrites (Cronin and Pizzarello, 1997; Pizzarello and Cronin, 2000; Pizzarello et al. 2008; Glavin et al. 2009; Myrgorodska et al. 2015).

Considerable progress has been made toward understanding interstellar photosynthetic and photolytic processes via laboratory experiments mimicking the induction of asymmetry in prebiotic molecules, particularly amino acids. Cometary ice evolution experiments demonstrated that amino acids including proteinogenic, nonproteinogenic, and diamino acids form from representative interstellar/protostellar $\mathrm{C}_{1}$ - and $\mathrm{N}_{1}$-unit molecules (Muñoz Caro et al. 2002, Bernstein et al. 2002, Meinert et al. 2012a) when subjected to Lyman- $\alpha$ radiation at very low temperatures. Experimental attempts for further asymmetric synthesis of amino and diamino acids from an astrophysically relevant achiral ice mixture containing methanol, water and ammonia by means of circularly polarized synchrotron radiation (CPSR) revealed the asymmetrical formation of up to five amino acids - alanine, 2-aminobutyric acid (2-Aba), valine, norvaline, and 2,3-diaminopropionic acid - with a maximum $e e_{\mathrm{L}}$ of $-2.54 \%$ for 2-Aba (Modica et al. 2014; de Marcellus et al. 2011). The induced enantiomeric enrichments were shown to depend on the helicity of the CPSR and were proportional to the number of chiral photons per deposited ice molecule. Moreover, the measured $e e_{\mathrm{L}}$ in the five amino acids were of the same sign for a given helicity (Modica et al. 2014). These experiments suggest that molecules of prebiotic interest are formed in inter/protostellar environments where they might have been subjected to CP vacuum-UV radiation before their arrival on early Earth via comets, meteorites and/or interstellar dust particles (Oró 1961; Chyba et al. 1990). This scenario is probed by the ambitious European Space Agency's Rosetta spacecraft investigating in situ comet 67P/Churyumov-Gerasimenko (67P/C-G). The imaging of the nucleus accomplished to date has revealed a smallish sized comet of average diameter $4 \mathrm{~km}$, rotating with a period of 12.7 hours. Further results of the mission as they emerge will be watched with eager anticipation, in particular for signs of any connections that might relate to the molecular and stereochemical origins of life (Meierhenrich 2015). Among the instruments that are examining the surface and environment of 67P/C-G's nucleus in unprecedented detail, the COSAC (Cometary Sampling and Composition) instrument aboard Rosetta's Philae lander is focusing on the enantioselective analysis of chiral organic molecules including amino acids.

Whether all amino acids are asymmetrically formed and/or photolysed at the same energy of incident CPL and whether the sign of the ee induced is the same for (at least) all proteinogenic amino acids in cometary matter can be understood through asymmetric photolysis experiments using circularly polarized synchrotron radiation. Given that CPL-mediated photochemistry depends on the circular dichroism (CD, $\Delta \varepsilon=\varepsilon_{\text {right }}-\varepsilon_{\text {left }}$ ) of the reactants, i.e. the differences in the extinction coefficients of right-and left-handed CPL (Inoue \& Ramamurthy 2004), the knowledge of CD spectra of amino acids in their amorphous solid state over a large spectral range is crucial for such experiments (Meierhenrich et al. 2010). Studying the chiroptical properties of amino acids in the isotropic solid state is assumed to best represent the interstellar/protostellar conditions in which amino acids are thought to be formed and/or altered. Moreover, an extended spectral region with possible electronic transitions in the vacuum UV range is accessible by eliminating the presence of water or any organic solvent commonly used in CD measurements. Rather than recording CD spectra alone, Meinert et al. (2012b) have introduced anisotropy spectroscopy $g(\lambda)$ that measures the ratio between the differential extinction coefficient $\Delta \varepsilon$ and the extinction coefficient $\varepsilon$ allowing for the wavelength-dependent determination of ees inducible into 
chiral organic molecules by asymmetric photolysis with circularly polarized light. This work has emphasized - as have earlier works (Kuhn and Braun 1929; Balavoine et al. 1974; Flores et al. 1977) the importance of the anisotropy factor $g(g=\Delta \varepsilon / \varepsilon)$ in controlling the enantiomeric purity of the products in asymmetric photochemical processes. Based on these newly recorded anisotropy spectra, first energy-dependent asymmetric photolysis experiments have been recently performed (Meinert et al. 2014a).

Herein we report on anisotropy-guided CPL irradiation experiments of racemic alanine films using far-UV circularly polarized synchrotron radiation at the beam line DESIRS, SOLEIL (France). To theoretically support our anisotropy measurements of isotropic alanine enantiomers and assign the corresponding ECD spectrum down to $130 \mathrm{~nm}$, we have calculated the anisotropy factor $g$ as function of the wavelength for zwitterionic L-alanine using time-dependent density functional theory (TDDFT). The obtained $g$-spectra of three particular low energy conformations reproduced the peak positions and intensities of the experimentally observed spectrum, thereby elucidating the detailed structure of zwitterionic L-alanine in the isotropic solid state. Exposure of racemic alanine to optimal circularly polarized photon energies predicted by $g(\lambda)$ yielded the second highest enantiomeric enhancement yet reported for an asymmetric photolysis of an amino acid. The induced ee is shown to depend on the photolysis rate, the wavelength and the helicity of the CPSR. Such wavelengthdependence of asymmetric photochemical reactions may provide insight into the abiotic origins of homochirality in living matter by means of interstellar circularly polarized photons.

\section{Materials and Methods}

\section{Anisotropy spectra}

Anisotropy spectra of alanine enantiomers were recorded with a new experimental set-up using the CD1 beam line on ASTRID (Evans et al. 2013) at Aarhus University (Denmark) prior to irradiation experiments. Isotropic films of alanine were condensed onto $\mathrm{MgF}_{2}$ optical windows in vacuum using a custom-built temperature- and pressure-controlled ultra-high vacuum (UHV) sublimation chamber (Meierhenrich et al. 2010). The extinction $\varepsilon$ and its corresponding differential extinction coefficient $\Delta \varepsilon$ in the vacuum UV and UV spectral range of the films were measured simultaneously to obtain $g(\lambda)=\Delta \varepsilon / \varepsilon$. Details on the sublimation and recording of anisotropy spectra are given in Meinert et al. (2012b).

For the theoretical calculations of anisotropy spectra of zwitterionic alanine, the rotational strength $R$ and dipole strength $D$ were calculated using time dependent density functional theory (TDDFT) with the B3P86 hybrid functional in combination with the $6-31+G(d, p)$ basis set as in Meinert et al. (2014a). In this previous calculation vertical anisotropy spectra were obtained first then treated for simulated line broadening effects. However to more closely parallel the derivation of experimental anisotropy spectra, we now obtain the absorption and ECD vertical spectra first, then simulate line broadening effects and finally form theoretical anisotropy spectra. This allowed us to overcome the numerical difficulties due to quasi-singular behaviors of the $g$-factor, (which, like Born-Oppenheimer potential energy surfaces (Cassam-Chenaï 2006), is not an observable but a ratio of observables), and to extend our predicted spectra down to about $90 \mathrm{~nm}$. The bond lengths and bond angles of zwitterionic L-alanine given in (Kitayama 2002) were used in all our calculations, and we only varied the angle, $\Phi$, of the plane of the carboxylate $\left(\mathrm{COO}^{-}\right)$group with respect to the $\mathrm{C}-\mathrm{C}-\mathrm{N}$-plane and the torsion angle, $\theta$, of the ammonium $\left(\mathrm{NH}_{3}{ }^{+}\right)$group. For the former angle eight different values were used in our study, while for the latter there were two values: $\theta=0^{\circ}$ corresponding to a staggered conformation and $\theta=$ $60^{\circ}$ corresponding to an eclipsed conformation with respect to the C-atom of the carboxylate group. For each nuclear geometry, characterized by the $(\Phi, \theta)$ values, the first 200 excited states above the ground state (i.e. all electronic states up to about $84 \mathrm{~nm}$ depending on the geometry) were calculated by TDDFT as implemented in the Gaussian 03 computer code (Gaussian, Inc. 2004). Ideally, line broadening of absorption and ECD spectra should be performed by using a theoretical modelling of vibrational effects such as the method developed by Santoro et al. (Santoro 2008) which include Duschinski and Herzberg-Teller effects and which is available in Gaussian 09. However, given the number of electronic states and the resolution of the experimental spectra, we found it more appropriate to use an empirical Gaussian broadening: 
$f_{\Phi, \theta}(\lambda)=\sum_{i \leq 200} f_{i, \Phi, \theta}^{T D D F T} \frac{e^{-\left(\frac{\lambda-\frac{C_{e V \rightarrow n m}}{E_{i, \Phi, \theta}^{T D D F T}+0.4}}{12}\right)^{2}}}{\sqrt{12 \pi}}$,

where the wavelength, $\lambda$, is in $\mathrm{nm}$, and $f$ stands for either the dipole strength, $D$, closely related to the extinction) or the rotational strength, $R$, (closely related to the differential absorption). $\mathrm{C}_{\mathrm{eV} \rightarrow \mathrm{nm}} \sim 1239.84187$ is the conversion factor from energy in $\mathrm{eV}$ to wavelength in $\mathrm{nm}$. The summation extends over all TDDFT calculated electronic excited states, indexed by $i, f_{i, \Phi, \theta}^{T D D F T}$ denoting the TDDFT dipole strength or rotational strength (according to the meaning of $f$ ) associated with the transition from the ground state to the $i^{\text {th }}$ excited state. A width of $12 \mathrm{~nm}$ has been found suitable to compare the calculated with experimental spectra, and an empirically optimized shift of $0.4 \mathrm{eV}$ has been added to the transition energy in eV, denoted by $E_{i, \Phi, \theta}^{T D D F T}$, a value somewhat lower than that of $0.6 \mathrm{eV}$ previously used in (Kaneko 2009) and (Meinert et al. 2014a). Eventually, anisotropy spectra were obtained according to

$$
g_{\Phi, \theta}(\lambda)=\frac{4 R_{\Phi, \theta}(\lambda)}{D_{\Phi, \theta}(\lambda)}
$$

\section{Asymmetric photolysis}

The asymmetric photolysis experiments of solid-state alanine were carried out using VUV circularly polarized synchrotron radiation generated by the electromagnetic variable polarizing undulator (OPHELIE2, HU640 type) at the DESIRS beam line (Nahon et al. 2012) at the SOLEIL facility, France. The monochromator set at the zero ${ }^{\text {th }}$ order transmitted the intense $\left(\sim 10^{15}\right.$ photons/sec integrated flux) pseudo white light of the undulator (Gaussian shape with a $7 \%$ energy bandwidth) centered at a photon energy that can be tuned. Higher harmonics from the undulator were cut-off by a Xe-filled gas filter. Films of D,L-alanine-2,3- ${ }^{13} C_{2}$ (Sigma Aldrich) with a constant film thickness of $300 \mathrm{~nm}$ were prepared using the UHV sublimation-condensation chamber prior to irradiation. Scanning electron microscope images previously provided by Meinert et al. (2014a) confirmed the non-crystalline, amorphous solid state and isotropic orientation of alanine in the condensed phase. These amorphous rac-alanine films were individually irradiated with right ( $r$ )- and left-handed (I) CPSR at two different photon energies $(6.19 \mathrm{eV}$ and $6.74 \mathrm{eV})$ corresponding to $g(\lambda)$ extrema for 2.5 to $5 \mathrm{~h}$. The absolute circular polarization rates, as measured by an in situ dedicated polarimeter, were above $97 \%$ for both helicities. During synchrotron irradiation the transmission of CP light was followed by a dedicated in-vacuum movable XUV photodiode.

\section{GCXGC analysis of irradiated rac-alanine films}

Irradiated and non-irradiated reference samples were extracted from the VUV-MgF 2 optical window with $3 \times$ $8.3 \mu \mathrm{L}$ water (HPLC grade) followed by $3 \times 8.3 \mu \mathrm{L} 0.2 \mathrm{M} \mathrm{HCl}$ and transferred into $1 \mathrm{~mL}$ conical reaction vials (Wheaton). The extracted samples were derivatized to form $N$-ethoxycarbonyl heptafluorobutyl esters (ECHFBE), adding first a mixture of $25 \mu \mathrm{L}$ of heptafluoro-1-butanol (Alfa Aesar) and pyridine (3:1, v/v) followed by $5 \mu \mathrm{L}$ of ethyl chloroformate (Sigma Aldrich). The derivatives were extracted with $20 \mu \mathrm{L}$ of chloroform and transferred into GC vials equipped with $100 \mu \mathrm{L}$ inserts. The samples were then analyzed by enantioselective two-dimensional gas chromatography coupled to a time-of-flight mass spectrometer (GCXGC-TOFMS) using a LECO Pegasus 4D instrument (Meinert et al. 2014b). Aliquots of $1 \mu \mathrm{L}$, injected in splitless mode, were separated on a Chirasil-D-Val as first stationary column ( $24.85 \mathrm{~m} \times 0.25 \mathrm{~mm}$ ID, $0.08 \mu \mathrm{m}$ film thickness) modulator-coupled by a modulation time of $P_{\mathrm{m}}=2.5 \mathrm{~s}$ to a DB Wax secondary column ( $1.4 \mathrm{~m} \times 0.1 \mathrm{~mm} \mathrm{ID}, 0.1 \mu \mathrm{m}$ film thickness). Samples were alternately injected at least 3 times in order to accurately determine ee values with statistical error bars by software-based volume peak integration. A blank sample was also prepared and analyzed using the same analytical conditions to investigate any biological and/or laboratory ${ }^{13} \mathrm{C}$-sources. 


\section{Results and Discussion}

Prior to the asymmetric photolysis experiments at SOLEIL synchrotron, the anisotropy spectra $(g=\Delta \varepsilon / \varepsilon)$ of alanine enantiomers were recorded at ASTRID, ISA, Aarhus University. Using an ultra high vacuum (UHV) sublimation-condensation chamber, amorphous films of defined thickness of enantiopure L- and D-alanine were deposited onto VUV optical windows. Anisotropy spectra of these isotropic films were recorded between 130 and $330 \mathrm{~nm}$ using a synchrotron light source which allows $\Delta \varepsilon$ and $\varepsilon$ to be determined with high precision over the entire spectral range. One of the main difficulties in studying the wavelength dependence of the anisotropy factor $g$, is to determine the absorption coefficient $\varepsilon$ above $220 \mathrm{~nm}$ because of its weak intensity and scattering effects, which induce large errors in the anisotropy spectrum. Figure 1a shows the $g$-spectra of L- and D-alanine enantiomers. The anisotropy spectrum of L-alanine (black line) is characterized by two major extrema: a maximum at $184 \mathrm{~nm}\left(g_{184}=0.0061\right)$ and a minimum at $200 \mathrm{~nm}\left(g_{200}=-0.083\right)$. As expected, the anisotropy spectrum of D-alanine (red line) is an almost perfect mirror spectrum of L-alanine, i.e. the anisotropy factor $g$ is nearly identical in magnitude but opposite in sign in the recorded wavelength range. We have recently simplified the kinetic resolution of CPL-induced photodecomposition of amino acids considered by Kagan and coworkers (Balavoine et al. 1974) and found that the ee value as a function of $g$ and the extent of reaction $\xi$ in the low $g$ limit becomes:

$$
e e \geq\left(1-(1-\xi)^{g / 2}\right) \times 100 \%
$$

(Meinert et al. 2012b). This expression was used to estimate the ee values which can be photochemically induced into rac-alanine by asymmetric photochemical irradiation at three selected photolysis rates $(\xi=99.99$, 99.80 , and $99.50 \%$ ) as a function of $g$ (Fig. 1a, dotted lines).
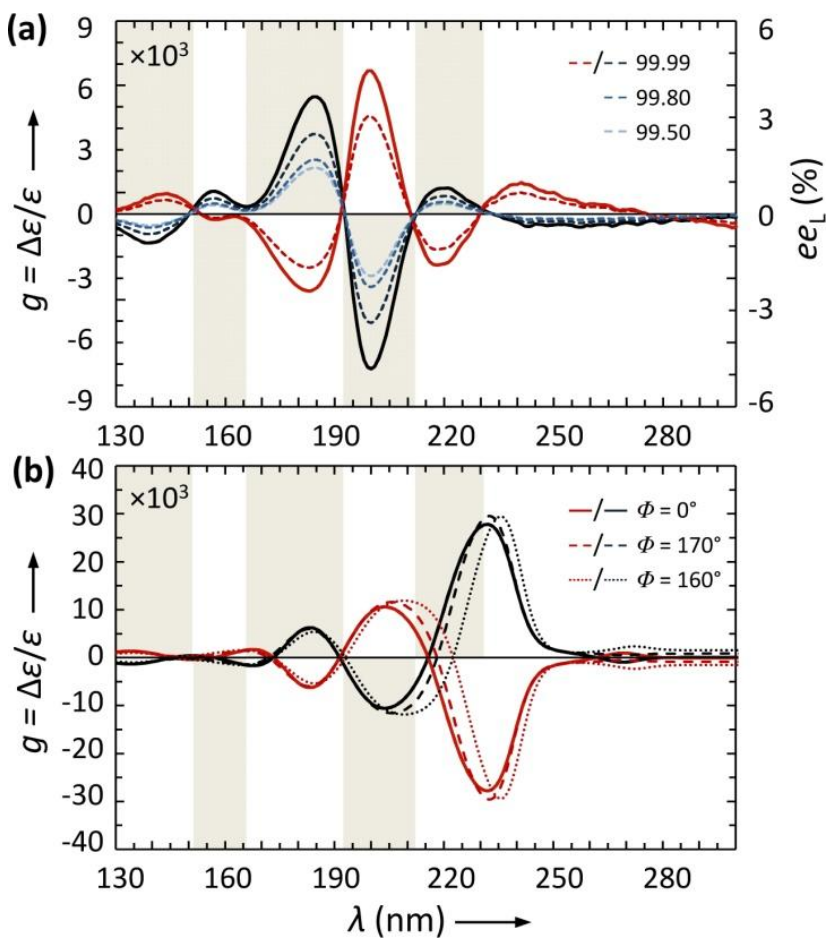

Fig. 1 Anisotropy spectra $g(\lambda)$ of alanine in the vacuum UV and UV spectral range. (a) Anisotropy spectra (thick lines) of enantiopure L-alanine (black) and D-alanine (red) condensed as isotropic amorphous films. Dotted lines represent the predicted L-enantiomeric excess (\%ee $\mathrm{L}_{\mathrm{L}}$ ) induced by either left or right-CPL as a function of $g$ and preselected values of the extent of reaction $\xi$ of $99.99,99.80$, and $99.50 \%$. (b) Calculated anisotropy spectra using time dependent density functional theory of zwitterionic L-alanine (black) and D-alanine (red) for three different nuclear geometries varying the internal rotation angle $\Phi$, which corresponds to the angle between the plane of the carboxylate group with respect to the $\mathrm{C}-\mathrm{C}-$ $\mathrm{N}$-plane and a constant torsion angle $\theta=60^{\circ}$ of the ammonium group.

Anisotropy spectra, although not directly observable, are interesting quantities for a comparison between theory and experiment, since, in a fashion very similar to flipping ratios formed in polarised neutron diffraction (Cassam-Chenaï 1996), they emphasize the property probed in the experiment, that is circular dichroism in the present case. In Fig. 2, we display theoretical anisotropy spectra corresponding to different nuclear geometries, i.e. conformers. Clearly, the anisotropy factor is very sensitive to the two geometric parameters: the internal rotation angles $(\Phi, \theta)$. Comparison with experiment Fig. 1a reveals that among all the calculated spectra only 
three agree satisfactorily in a qualitatively way with the experimental spectrum. Interestingly, the three corresponding geometries, namely $\left(160^{\circ}, 60^{\circ}\right),\left(170^{\circ}, 60^{\circ}\right),\left(180^{\circ}=0^{\circ}, 60^{\circ}\right)$, also happen to have the lowest calculated energies. These three predicted $g(\lambda)$ curves are displayed in the range between 130 and $300 \mathrm{~nm}$ in Fig. 1b. For better comparison, the coloured background sections in Fig. 1a are included in Fig. 1b. The peaks at 184 and $200 \mathrm{~nm}$ are well reproduced, the $g$-factor extremal values being only slightly overshot. The TDDFT values of the $g$-factor are $g_{184}=0.0087$ and $g_{200}=-0.0126$ for L-alanine at the $\left(160^{\circ}, 60^{\circ}\right)$ geometry and $g_{184}=$ 0.0113 and $g_{200}=-0.0144$ at $\left(0^{\circ}, 60^{\circ}\right)$. The largest peak at $220 \mathrm{~nm}$ in the theoretical calculation is much higher than its experimental counterpart. However, this feature is an average and, as can be seen from Fig. S2 of Meinert et al. (2014a), it is very dependent upon the film considered. So, the calculated spectra seem reliable and strongly hint to an alanine geometry in the film quenched around the eclipsed ammonium conformation with the carboxylate group being in or close to the CCN plane.

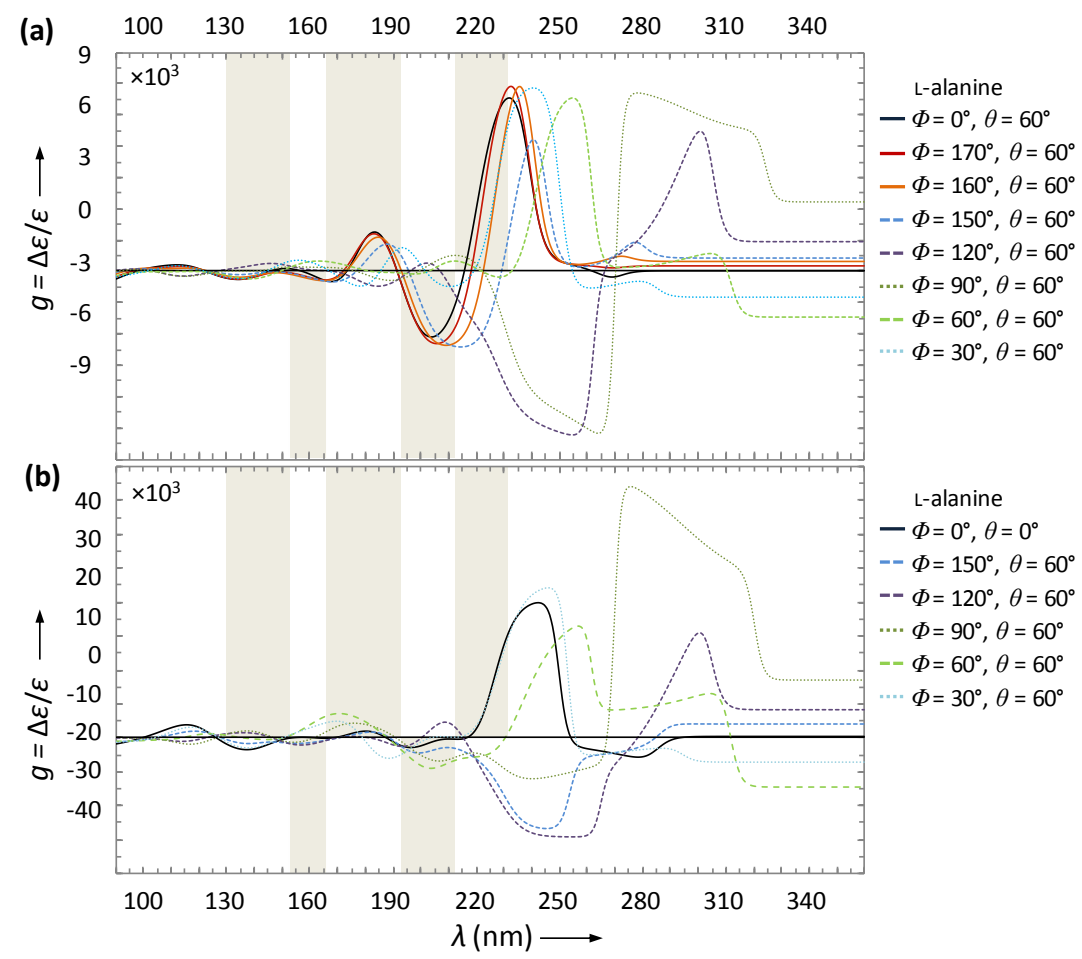

Fig. 2 Comparison of time dependent density functional theory calculated anisotropy spectra corresponding to different carboxylate group torsion angles $\left(\Phi=0,-30,-60,90,60\right.$, and $\left.30^{\circ}\right)$ and for (a) staggered ammonium group conformations $\left(\theta=60^{\circ}\right)$, as well as (b) eclipsed ammonium group conformations $\left(\theta=0^{\circ}\right)$, with respect to the $\mathrm{C}$-atom of the carboxylate group.

Based on the anisotropy spectra, the optimal wavelength of CPL to induce high ees into isotropic films of racalanine via enantioselective photolysis were determined to be 184 and $200 \mathrm{~nm}$. At $g_{200}$, an ee of about $4 \%$ can potentially be photochemically induced into rac-alanine, assuming the extent of reaction $\xi=0.9999$ is reached. The anisotropy spectra of alanine further indicates the generation of opposite enantiomeric enhancement for a given helicity of incident CPL at $g_{200}$ and $g_{184}$ as well as photochemically inactive wavelengths due to several zero-crossings in the recorded anisotropy.

In order to experimentally confirm the wavelength-dependence of CPL-induced asymmetry into chiral molecules, we photolysed racemic alanine with quasiperfect circularly polarized synchrotron radiation at the VUV beam line at the SOLEIL facility. In this study, three isotropic amorphous rac-alanine films were systematically irradiated with CPSR varying $i$ ) the polarization and ii) the wavelength, as summarized in Table 1. Racemic alanine films with a thickness of $300 \mathrm{~nm}$ were prepared under vacuum, sublimating and re-condensing ${ }^{13} \mathrm{C}$-labeled $\mathrm{D}, \mathrm{L}$-alanine on $\mathrm{MgF}_{2}$ windows. Two isotropic amorphous rac- ${ }^{13} \mathrm{C}$-alanine samples were individually 
subjected to $l$ - and $r$-CPSR at $\lambda=184 \mathrm{~nm}$. With the help of a non-irradiated $300 \mathrm{~nm}$ rac-alanine film (Table 1, reference sample 3 ), the total photolysis rates $(\xi)$ were determined to be $99.88 \%$ for sample $\mathrm{C} 1$ irradiated with I-CPSR and $99.87 \%$ for sample C2 irradiated with $r$-CPSR, respectively. The enantioselective GCXGC-TOFMS analysis revealed an L enantiomeric enrichment of $1.66 \%$ in sample C2 (Fig. 3a). As expected, the irradiation of sample C1 at $g_{184}$ with the opposite handedness of incident CPSR was found to induce an equivalent excess of the $\mathrm{D}$-alanine enantiomer (Fig. 3b). The multidimensional gas chromatographic analysis of sample $\mathrm{C} 1$ yielded an $e e_{\mathrm{L}}$ value of $-1.95 \%$
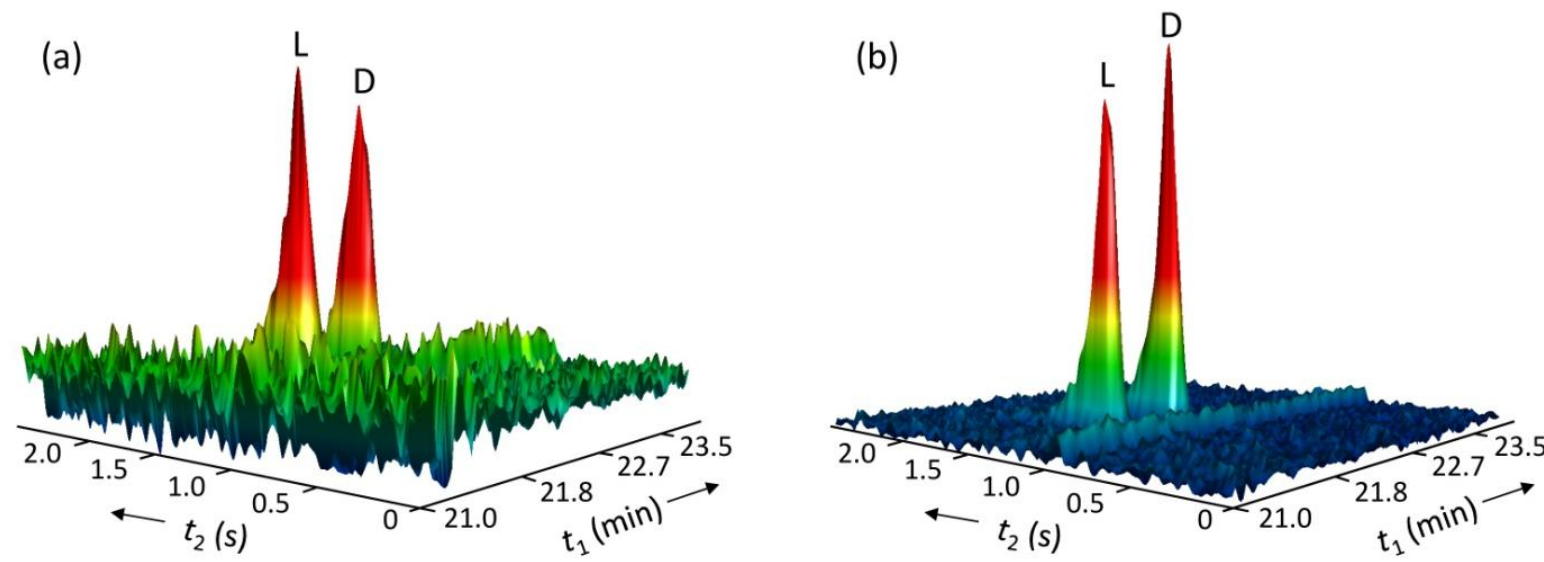

Fig. 3 Close-up of the two-dimensional enantioselective gas chromatographic resolution of ${ }^{13} \mathrm{C}$-alanine enantiomers after irradiation with circularly polarized light (cpl) at anisotropy extrema $6.74 \mathrm{eV}(184 \mathrm{~nm})$. Isotropic films of racemic ${ }^{13} \mathrm{C}$-alanine were individually irradiated with (a) $r$-cpl for $5 \mathrm{~h}$, resulting in an ee of L-alanine and (b) $\mathrm{l}$-cpl for $4 \mathrm{~h}$, resulting in an equivalent ee of D-alanine.

A third sample, D1, was irradiated for 3.5 hours at $200 \mathrm{~nm}$ using $r$-CPSR, with its photolytic decomposition resulting in a reversal in the sign of induced $e e_{\mathrm{L}}$ of $-4.11 \%$, compared to the positive ee induced in the similarly $r$-CPSR irradiated $\mathrm{C}_{2} \mathrm{~g}_{185}$ sample (Table 1). These experimental data confirm our previously obtained results on the wavelength- and polarization-dependence of the asymmetrical photodegradation of rac-alanine films (Meinert et al. 2014a), and are all summarized in Table 1. Due to limited beam time at the SOLEIL synchrotron, we were unable to carry out the necessary confirmation of the $-4.11 \%$ enantiomeric enhancement reported in this study by the equal but opposite ee induced into rac-alanine with opposite-handed synchrotron radiation, i.e. I-CPSR. However, our previous data, in particular sample set $\mathrm{A} 1$ and $\mathrm{A} 2$, provide evidence for the reliability of the $e e_{\mathrm{L}}$ found in sample D1.

The evolution of the absolute $e e$ as a function of the extent of reaction $\xi$ in the asymmetric photodecomposition of racemic alanine by CPL at $\lambda=184$ and $200 \mathrm{~nm}$ is shown in Fig. 4. These curves demonstrate that high enantiomeric enrichments can be attained by asymmetric photodecomposition if significant photolysis rates are reached and that higher values of $g$ should yield an almost optically pure residue before the decomposition of the starting material has gone to completion. Overall, our experimental CPSRinduced ee values show good agreement with the predicted ee-curves. For both wavelengths, the experimentally induced enantiomeric excesses are somewhat higher than those predicted by Equation (2) at $\xi>$ 99.9\%, whereas lower optical purities are observed in our experiments at $\xi<99.8 \%$ as predicted by the anisotropy factors (with $g_{184}=0.0055$ and $g_{200}=-0.007$ ). This observation suggests that the present asymmetric photolysis of rac-alanine may not strictly follow simple first-order kinetics upon which Equation (2) is derived. On the other hand, we cannot fully exclude experimental errors from causing this deviation: while the inaccuracies of the absolute magnitude of the induced ee values are given as \pm error bars in Fig. 4 , we are unable to provide the corresponding errors for the photolysis rates $\xi$ due to limited experimental data. Moreover, the predicted ee values (red and blue line, Fig. 4) are based on the anisotropy factors $g$ at 184 and $200 \mathrm{~nm}$, respectively; two experimentally obtained parameters using anisotropy spectroscopy. 
Table 1 Experimental and predicted enantiomeric excesses $e e_{\mathrm{L}}$ induced via asymmetric photolysis in isotropic films of racemic ${ }^{13} \mathrm{C}$-alanine as function of irradiation wavelength $\lambda$, helicity of circularly polarized synchrotron radiation (CPSR), irradiation time, and extent of reaction $\xi$.

\begin{tabular}{|c|c|c|c|c|c|c|c|}
\hline $\begin{array}{l}{ }^{13} \mathrm{C} \text {-alanine } \\
\text { sample }\end{array}$ & $\lambda[\mathrm{nm}]$ & $\begin{array}{l}\text { Circular } \\
\text { polarization }\end{array}$ & $\begin{array}{l}\text { Irradiation } \\
{[\mathrm{h}]}\end{array}$ & $\begin{array}{l}\text { Extent of } \\
\text { reaction } \xi[\%]\end{array}$ & $e e_{\mathrm{L}}[\%]^{\mathrm{a}}(n)^{\mathrm{b}}$ & $e e_{\mathrm{L}}[\%]^{\mathrm{C}}$ & reference \\
\hline A1 & 200 & I-CPSR & 5.0 & 99.95 & $4.19 \pm 0.14(6)$ & 3.10 & Meinert et al. 2014a \\
\hline$A 2$ & 200 & $r$-CPSR & 5.0 & 99.97 & $-4.22 \pm 0.21(5)$ & -3.42 & Meinert et al. 2014a \\
\hline A3 & 200 & I-CPSR & 2.5 & 99.56 & $0.57 \pm 0.17(9)$ & 2.22 & Meinert et al. 2014a \\
\hline A4 & 200 & $r$-CPSR & 2.5 & 99.66 & $-1.11 \pm 0.16(9)$ & -2.38 & Meinert et al. 2014a \\
\hline B1 & 184 & I-CPSR & 5.0 & 99.93 & $-3.12 \pm 0.34(5)$ & -2.24 & Meinert et al. 2014a \\
\hline B2 & 184 & $r$-CPSR & 5.0 & 99.96 & $3.15 \pm 0.30(4)$ & 2.36 & Meinert et al. 2014a \\
\hline B3 & 184 & I-CPSR & 2.5 & 99.89 & $-2.88 \pm 0.28(7)$ & -2.10 & Meinert et al. 2014a \\
\hline B4 & 184 & $r$-CPSR & 2.5 & 99.80 & $1.58 \pm 0.15(9)$ & 1.88 & Meinert et al. 2014a \\
\hline $\mathrm{C} 1$ & 184 & I-CPSR & 4.0 & 99.88 & $-1.95 \pm 0.35(5)$ & -2.06 & this study \\
\hline $\mathrm{C} 2$ & 184 & $r$-CPSR & 5.0 & 99.87 & $1.66 \pm 0.35(3)$ & 2.00 & this study \\
\hline D1 & 200 & $r$-CPSR & 3.5 & 99.98 & $-4.11 \pm 0.32(3)$ & -3.59 & this study \\
\hline $\operatorname{Ref} 1^{d}$ & - & - & - & - & $0.08 \pm 0.15(7)$ & - & Meinert et al. 2014a \\
\hline $\operatorname{Ref} 2^{d}$ & - & - & - & - & $0.05 \pm 0.19(10)$ & - & Meinert et al. 2014a \\
\hline $\operatorname{Ref} 3^{d}$ & - & - & - & - & $0.10 \pm 0.11(5)$ & - & this study \\
\hline
\end{tabular}

${ }^{a}$ Experimentally determined by enantioselective GCxGC-TOFMS. The L-excess is defined as ee $e_{\mathrm{L}}=(\mathrm{L}-\mathrm{D}) /(\mathrm{L}+\mathrm{D})$, with a negative value corresponding to a D-excess. The data are mean values given with their standard deviation of $1 \sigma$.

${ }^{\mathrm{b}}$ Number of replicate analyses.

${ }^{\mathrm{c}}$ Predicted $e e_{\mathrm{L}}$ based on anisotropy factor $g$ and experimentally reached extent of reaction $\xi$; $e e=\left(1-(1-\xi)^{g / 2}\right)$ $\times 100 \%$.

${ }^{d}$ Non-irradiated sample: control and reference sample to calculate photolysis rate $\xi$.

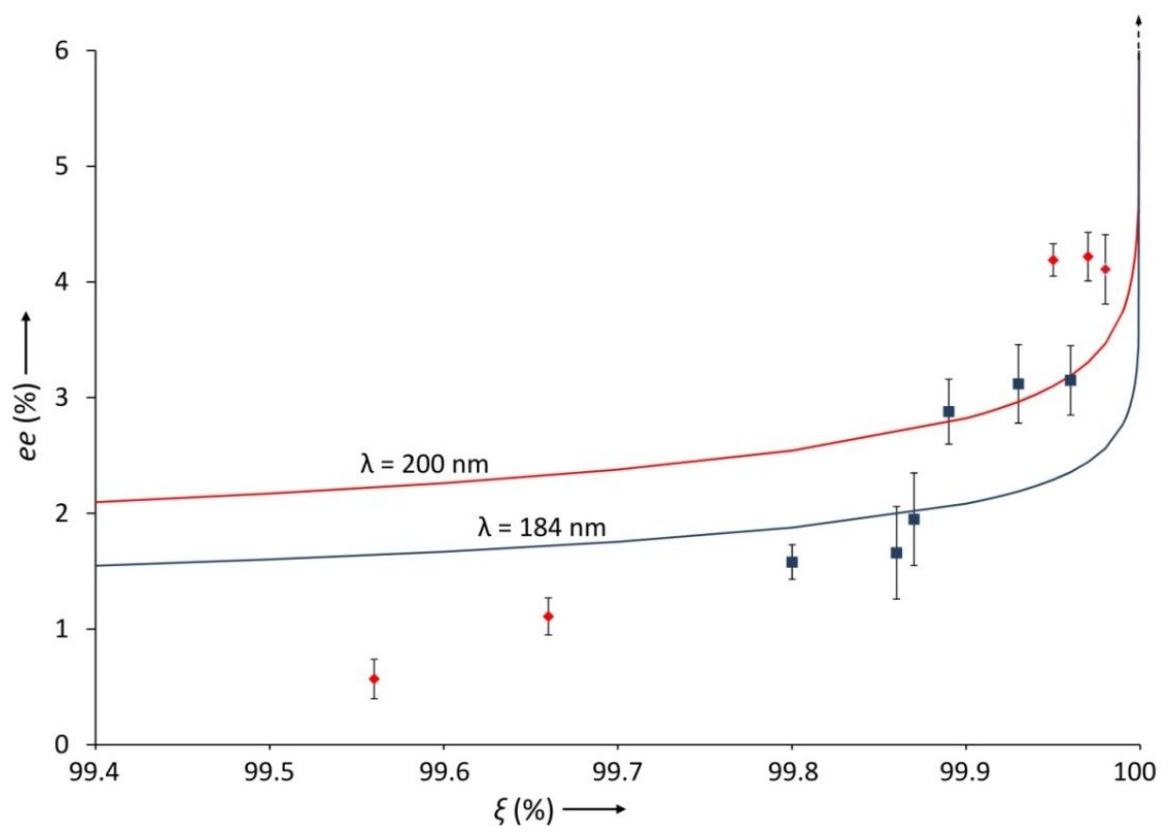

Fig. 4 Kinetic photoresolution of alanine by circularly polarized light (cpl). Rectangles are experimental data obtained by asymmetric photodestruction of racemic alanine in the solid state at cpl wavelength $\lambda=200 \mathrm{~nm}$ (red) and $184 \mathrm{~nm}$ (blue) as a function of the extent of reaction $\xi$. Curves are representing the calculated absolute values of the enantiomeric enhancement $(e e)$ based on experimentally determined anisotropy factors $g_{200}$ and $g_{184}$ as a function of $\xi$. 
In summary, anisotropy spectroscopy has been used to pinpoint the most favourable wavelengths for asymmetric excitation, and subsequent photoreactive photodecomposition of alanine. It further provides a direct means for quantifying how large the asymmetric effect of the various wavelengths of CPL might be in prebiotic molecules. Our anisotropy-guided experiments using the example of alanine have proven that both the magnitude and the sign of inducible ee depend on the wavelength and the helicity of the incident CPL in asymmetric photochemical reactions. Such wavelength-dependent stereocontrol is relevant for understanding the interstellar/protostellar origins of enantiomeric enrichments in prebiotic molecules. Given that asymmetric photolysis experiments have frequently been conducted in the context of protostellar ice simulations (Evans et al. 2012), it is reasonable to postulate that this sort of anisotropic chiral irradiation bias occurred in the molecular cloud that later collapsed to form our Solar System, where it has induced initial enantiomeric enrichments in prebiotic precursor molecules. Natural sources of CPL, however, are spectrally broadband, and as a consequence could lead to a competition in the sign of asymmetrically photolysed chiral molecules and/or in the rare event yield a zero integral of the multiple effective $C D$ bands over the entire spectral range according to the Kuhn-Condon rule (Mason 1997). However, the spectral energy distribution (SED) of the more massive stars that are associated with high degrees of CPL in star-forming regions (Chrysostomou et al. 2007) favours high-energy photons during their earlier stages of evolution (Robitaille et al. 2006). This may lead to a non-zero effective anisotropy and ultimate optical yields in chiral precursors.

The hypothesis of photochemical asymmetry generation by means of circularly polarized radiation in protostellar environments will be investigated by ESA's ongoing cometary Rosetta mission (Meierhenrich 2015). Comets hold many secrets about the birth and evolution of the Solar System, and about the origin and history of many molecules, including chiral molecules that are fundamental for life on Earth. Now that Rosetta has reached comet 67P/Churyumov-Gerasimenko (67P/C-G), research is on course to probe the cometary surface to unlock this icy treasure chest. Rosetta's lander Philae detached from the Rosetta orbiter in November 2014 is equipped with the cometary sampling and composition instrument COSAC (Goesmann et al. 2007; Goesmann et al. 2014). COSAC uses eight parallel capillary columns with different stationary phases coupled to a time-of-flight mass spectrometer to analyze organic molecules in situ in the ices of the nucleus of comet 67P/C-G. Rosetta is the first space mission ever that is equipped with a chirality evaluation device. COSAC's five achiral stationary phases (Szopa et al. 2014) and the three chiral stationary phases (Meierhenrich et al. 2013) have been tested successfully for long term vacuum exposure. Particular interest has been given to the chirality experiment, by which COSAC will separate, quantify, and identify chiral organic molecules in cometary ices (Myrgorodska et al. 2015). COSAC's selected chiral stationary phases are complementary, i.e., suitable for the enantiomer resolution of many families of chiral organic molecules including hydrocarbons (Meierhenrich et al. 1999, 2001a, 2003), amines (Meierhenrich et al. 1999), alcohols including diols (Meierhenrich et al. 1999, Thiemann et al. 2001), carboxylic acids (Meierhenrich et al. 2001b), and amino acids (Thiemann and Meierhenrich 2001). COSAC data on the distribution of various chiral molecules and their enantiomeric excesses in cometary ices will provide a better understanding of the original chirality-inducing driving force behind enantiomeric enhancements in prebiotic samples.

\section{Acknowledgements}

We are grateful to the general technical staff of the ASTRID synchrotron and for technical and beam line assistance at the SOLEIL facilities. This work was supported by the Agence Nationale de la Recherche (ANR-07BLAN-0293 and ANR-12-IS07-0006). This work was furthermore supported by the 13 Integrated Activity on Synchrotron and Free Electron Laser Science (IA-SFS), contract no. RII3-CT-2004-506008, under the Research Infrastructure Action of the FP6 EC program Structuring the European Research Area as well as the European Community's Seventh Framework Program (FP7/2007-2013; grant no. 226716).

\section{Conflicts of Interest}

All authors are requested to disclose any actual or potential conflict of interest including any financial, personal or other relationships with other people or organizations 


\section{References}

Bailey J, Chrysostomou A, Hough JH, Gledhill TM, McCall A, Clark S, Ménard F, Tamura M (1998) Circular polarization in star-formation regions: Implications for biomolecular homochirality. Science 281:672-674

Balavoine G, Moradpour A, Kagan HB (1974) Preparation of chiral compounds with high optical purity by irradiation with circularly polarized light, a model reaction for the prebiotic generation of optical activity. J Am Chem Soc 96:5152-5158

Bernstein MP, Dworkin JP, Sandford SA, Cooper GW, Allamandola L (2002) Racemic amino acids from the ultraviolet photolysis of interstellar ice analogues. Nature 416:401-403

Brack A (1998) The Molecular Origins of Life - Assembling the Pieces of the Puzzle. Cambridge University Press, Cambridge

Cassam-Chenaï P, Wolff SK, Chandler GS, Figgis BN (1996) Ensemble-representable densities for atoms and molecules. II. Application to $\mathrm{CoCl}_{4}^{-}$. Int J Quantum Chem 60:667-680

Cassam-Chenaï P (2006) On non-adiabatic potential energy surfaces. Chem Phys Lett 420:354-357

Chrysostomou A, Lucas PW, Hough JH (2007) Circular polarimetry reveals helical magnetic fields in the young stellar object HH 135-136. Nature 450:71-73

Chyba CF, Thomas PJ, Brookshaw L, Sagan C (1990) Cometary delivery of organic molecules to the early Earth. Science 249:366-73

Cronin JR, Pizzarello S (1997) Enantiomeric excesses in meteoritic amino acids. Science 275:951-955

De Marcellus P, Meinert C, Nuevo M, Filippi J-J, Danger G, Deboffle D, Nahon L, d'Hendecourt L, Meierhenrich UJ (2011) Non-racemic amino acid production by UV irradiation of achiral interstellar ice analogs with circularly polarized light. Astrophys J Lett 727:L27 (6pp)

Evans AC, Meinert C, Bredehöft JH, Giri C, Jones NC, Hoffmann SV, Meierhenrich UJ (2013) Anisotropy spectra for chiral differentiation of biomolecular building blocks. Top Curr Chem 341:271-300

Evans AC, Meinert C, Giri C, Goesmann F, Meierhenrich UJ (2012) Chirality, photochemistry and the detection of amino acids in interstellar ice analogues and comets. Chem Soc Rev 41:5447-5458

Flores JJ, Bonner WA, Massey GA (1977) Asymmetric photolysis of (RS)-leucine with circularly polarized ultraviolet light. J Am Chem Soc 99:3622-3625

GAUSSIAN03 (2004) Gaussian, Inc., Wallingford CT

Glavin DP, Dworkin JP (2009) Enrichment of the amino acid L-isovaline by aqueous alteration on $\mathrm{Cl}$ and $\mathrm{CM}$ meteorite parent bodies. Proc Natl Acad Sci 106:5487-5492

Goesmann F, Rosenbauer H, Roll R, Szopa C, Raulin F, Sternberg R, Israel G, Meierhenrich UJ, Thiemann W, Muñoz Caro GM (2007) COSAC, The cometary sampling and composition experiment on Philae. Space Sci Rev 128:257-280

Goesmann F, Raulin F, Bredehöft JH, Cabane M, Ehrenfreund P, MacDermott AJ, McKenna-Lawlor S, Meierhenrich UJ, Muñoz Caro GM, Szopa C, Sternberg R, Roll R, Thiemann WHP, Ulamec S (2014) COSAC prepares for sampling and in situ analysis of cometary matter from comet 67P/Churyumov-Gerasimenko. Planet Space Sci 103:318-330

Inoue Y, Ramamurthy V (2004) Chiral Photochemistry. Marcel Dekker, New York

Kaneko F, Yagi-Watanabe K, Tanaka M, Nakagawa K (2009) Natural circular dichroism spectra of alanine and valine films in vacuum ultraviolet region. J Phys Soc Jpn 78:013001

Kitayama T, Kiyonaga H, Morihashi K, Takahashi O, Kikuchi O (2002) Ab initio spin-orbit coupling SCF calculation of parity-violating energy of chiral molecules. J Mol Struct, Theochem 589-590:183-193

Kuhn W (1929) Quantitative relationships for natural optical activity. Phys Chem B 4:14-36

Kwon J, Tamura M, Hough JH, Kusakabe N, Nagata T, Nakajima Y, Lucas PW, Nagayama T, Kandori R (2014) Near-infrared circular polarization survey in star-forming regions: correlations and trends. Astrophys J Lett 795:L16 (7pp)

Mason SF (1997) Extraterrestrial handedness. Nature 389:804 
Meinert C, de Marcellus P, Le Sergeant d'Hendecourt L, Nahon L, Jones NC, Hoffman SV, Bredehöft JH, Meierhenrich UJ (2011) Photochirogenesis: Photochemical models on the absolute asymmetric formation of amino acids in interstellar space. Phys Life Rev 8:307-330; 337-338.

Meinert C, Filippi J-J, de Marcellus P, Le Sergeant d'Hendecourt L, Meierhenrich UJ (2012a) N-(2Aminoethyl)glycine and amino Acids in interstellar ice analogues. ChemPlusChem 77:186-191

Meinert C, Bredehöft JH, Filippi J-J, Baraud Y, Nahon L, Wien F, Jones NC, Hoffmann SV, Meierhenrich UJ (2012b) Anisotropy spectra of amino acids. Angew Chem 124: 4562-4565; Angew Chem Int Ed 51:44844487

Meinert C, Hoffman SV, Cassam-Chenaï P, Evans AC, Giri C, Nahon L, Meierhenrich UJ (2014a) Photon-energycontrolled symmetry breaking with circularly polarized light. Angew Chem 126:214-218; Angew Chem Int Ed 53:210-214

Meinert C, Meierhenrich UJ (2014b) Derivatization and multidimensional gas-chromatographic resolution of $\alpha$-alkyl and $\alpha$-dialkyl amino acid enantiomers. ChemPlusChem 79:781-785

Meierhenrich UJ, Thiemann WH-P, Rosenbauer H (1999) Molecular Parity Violation via Comets? Chirality 11:575-582

Meierhenrich UJ, Thiemann WH-P, Goesmann F, Roll R, Rosenbauer H (2001a) Enantiomer separation of hydrocarbons in preparation of Rosetta's 'chirality-experiment'. Chirality 13:454-457

Meierhenrich UJ, Thiemann WH-P, Rosenbauer H (2001b) Pyrolytic methylation assisted Enantioseparation of Chiral Hydroxycarboxylic Acids. J Anal Appl Pyrol 60:13-26

Meierhenrich UJ, Nguyen M-J, Barbier B, Brack A, Thiemann WH-P (2003) Gas chromatographic separation of saturated aliphatic hydrocarbon enantiomers on permethylated $\beta$-cyclodextrin. Chirality 15:13-16

Meierhenrich UJ, Filippi J-J, Meinert C, Hoffmann SV, Bredehöft JH, Nahon L (2010) Photolysis of rac-leucine with circularly polarized synchrotron radiation. Chem Biodiversity 7:1651-1659

Meierhenrich UJ, Cason JRL, Szopa C, Sternberg R, Raulin F, Thiemann WH-P, Goesmann F (2013) Evaluating the robustness of the enantioselective stationary phases on the Rosetta mission against space vacuum vaporization. Adv Space Res 52:2080-2084

Meierhenrich UJ (2015) Comets and their origin - the tool to decipher a comet. Wiley-VCH, Weinheim

Modica P, Meinert C, deMarcellus P, Nahon L, Meierhenrich UJ, Le Sergeant d'Hendecourt L (2014) Enantiomeric excesses induced in amino acids by ultraviolet circularly polarized light irradiation of extraterrestrial ice analogs: A possible source of asymmetry for prebiotic chemistry. Astrophys J 788:79 (11pp)

Muñoz Caro GM, Meierhenrich UJ, Schutte WA, Barbler B, Segovia AA, Rosenbauer H, Thiemann WHP, Brack A, Greenberg JM (2002) Amino acids from ultraviolet irradiation of interstellar ice analogues. Nature 416:403-406

Myrgorodska I, Meinert C, Martins Z, Le Sergeant d'Hendecourt L, Meierhenrich UJ (2015) Molecular Chirality in Meteorites and Interstellar Ices, and the Chirality-Experiment Onboard ESA's Cometary Rosetta Mission. Angew Chem Int Ed 54:1402-1412

Nahon L, de Oliveira N, Garcia GA, Gil JF, Pilette O, Marcouille B, Lagarde F, Polack F (2012) DESIRS: a state-ofthe-art VUV beamline featuring high resolution and variable polarization for spectroscopy and dichroism at SOLEIL. J Synchrotron Radiat 19:508-520

Oró J. (1961) Comets and formation of biochemical compounds on primitive Earth. Nature 190:389-90

Pizzarello S, Cronin JR (2000) Non-racemic amino acids in the Murray and Murchison meteorites. Geochim Cosmochim Acta 64:329-338

Pizzarello S, Huang Y, Alexandre MDR (2008) Molecular asymmetry in extraterrestrial chemistry: Insights from a pristine meteorite. Proc Natl Ac Sci USA 105:7300-7304

Robitaille TP, Whitney AW, Indebetouw R, Wood K, Denzmore P (2006) Interpreting spectral enery distributions from young stellar objects. I. A grid of 200,000 YSO model SEDs. JApJS 167:256-285 
Santoro F, Lami A, Improta R, Bloino J, Barone V (2008) Effective method for the computation of optical spectra of large molecules at finite temperature including the Duschinsky and Herzberg-Teller effect: The $Q_{\mathrm{x}}$ band of porphyrin as a case study. J Chem Phys 128:224-311

Szopa C, Sternberg R, Coscia D, Goesmann F, Gomes R, Legrand S, Jerome M, Meierhenrich UJ, Raulin F (2014) Gas chromatography for in situ analysis of a cometary nucleus V. Study of capillary columns robustness submitted to long term reduced environmental pressure conditions. J Chromatogr A, 1368:211-216

Thiemann WH-P, Rosenbauer H, Meierhenrich UJ (2001) Conception of the 'Chirality-Experiment' on ESA's Mission ROSETTA to Comet 46P/Wirtanen. Adv Space Res 27:323-328

Thiemann WH-P, Meierhenrich UJ (2001) ESA Mission ROSETTA Will Probe for Chirality of Cometary Amino Acids. Orig Life Evol Biosph 31:199-210 